\title{
The Use of Interpreters in Medical Triage during a Refugee Mass-Gathering Incident in Europe
}

\author{
Lykourgos Christos Alexakis, MD, DTM, MSc; ${ }^{1}$ Anastasia Papachristou, MD, MSc, MBA; ${ }^{1}$ \\ Chiara Baruzzi, MD; ${ }^{2}$ Angeliki Konstantinou, $\mathrm{MD}, \mathrm{MSc}^{3}$
}

1. Médecins Sans Frontières, Athens, Greece

2. Médecins Sans Frontières, Rome, Italy

3. Anesthesiology Department, Hospital zum Heiligen Geist, Frankfurt am Main, Germany

\author{
Correspondence: \\ Lykourgos Christos Alexakis, MD, \\ DTM, MSc \\ Médecins Sans Frontières \\ Athens, Greece \\ E-mail: 1chalexakis@gmail.com
}

Conflicts of interest: none

Keywords: communication barriers; interpreters; mass gathering; refugees; triage

Abbreviations:

MSF: Médecins Sans Frontières

START: Simple Triage and Rapid Treatment

Received: October 15, 2016

Revised: January 14, 2017

Accepted: February 5, 2017

Online publication: July 31, 2017

doi:10.1017/S1049023X17006781

\begin{abstract}
Introduction: During a refugees' mass-gathering incident in Kos Island, Greece, Médecins Sans Frontières (MSF; Brussels, Belgium) teams provided emergency medical care. A case report of the event focusing on difficulties encountered by the interpreters during triage and emergency response was prepared.

Methods: Data collected during the event were reviewed from the patient's register and qualitative interviews were obtained from the MSF interpreters involved in the response. In addition, a description of the event and a literature review were included.

Results: Total consultations were 49 patients, mainly from Syria, with an average age of 25 years. During triage, 20 patients were tagged green with only minor injuries; 11 patients were tagged yellow, mostly due to heat exhaustion, but also a hypertensive crisis, a diabetic, a pregnant woman with abdominal pain, and a peptic ulcer exacerbation. The remaining 18 patients were tagged red and diagnosed with heat syncope, except from a case of epileptic seizures and an acute chest pain patient. Interpreters were insufficient in number to accompany each doctor and every nurse providing care during the event. In addition, they were constantly disturbed by both refugees and fellow medical team members demanding their service. Interpreters had to triage and prioritize where to go and for whom to interpret. Conclusion: Interpreters are an integral part of a proper refugee reception system. They should be included in authorities planning where mass gatherings of refugees are expected. Appropriate training may be needed for interpreters to develop skills useful in mass gatherings and similar prehospital settings in order to better coordinate with the medical team.
\end{abstract}

Alexakis LC, Papachristou A, Baruzzi C, Konstantinou A. The use of interpreters in medical triage during a refugee mass-gathering incident in Europe. Prehosp Disaster Med. 2017;32(6):684-687.

\section{Introduction}

Currently, Europe is one of the regions in the world receiving large numbers of refugees. From January 1st through August 14th, 2015, a total of 160,172 refugees and migrants entered Greece, which included 158,456 sea arrivals and 1,716 through its land border with Turkey. ${ }^{1}$

Following a refugees' mass-gathering event in Greece, during which Médecins Sans Frontières (MSF; Brussels, Belgium) teams decided to provide emergency medical care, although they were not responsible for or involved in the event organization which was done by the local authorities, the following questions were raised: (1) Were there any prior reports of triage incidents involving refugees where interpreters were used to overcome the language barrier? (2) What were the difficulties faced by the team, and interpreters in particular, during triage? (3) Is there a way to improve coordination between interpreters and medical teams in refugee mass-gathering events?

Answers to the above questions may be useful to take into account in authorities' disaster planning, particularly in areas receiving large numbers of newly arriving refugees. For this reason, a literature review, as well as a report of the event which included analysis of routinely collected data and qualitative feedback from the interpreters involved, was organized.

\section{Report}

Starting from March 2015, MSF provided primary medical and psychological care to refugees and migrants arriving in Dodecanese Islands in Greece, where no organized refugee reception system was in place at the time. 
On August 12th, 2015, an estimated population of 4,000 newly arrived Arabic-speaking refugees were gathered inside a football stadium in Kos Island by the local authorities in order to be registered by the police. Although MSF was not involved with the organization of the response to this event, the MSF logistics and coordination team assessed the situation and identified that there was a lack of shade cover, insufficient access to water and sanitation sites, and no medical service at the site. Therefore, MSF intervened in order to provide emergency medical care once refugees started collapsing.

The first part of an MSF medical team (one doctor, one nurse, and one Arabic interpreter) arrived at the scene at 11:00AM and initially organized a triage area under a sun umbrella. Subsequently, a treatment area was designated 30 meters away which consisted of two small storage rooms (about 20 and 10 square meters, respectively) and their yard (about eight by three meters).

Initially, triage and rapid clinical assessment were undertaken by the doctor acting both as triage and treatment leader. As the number of patients and workload increased, the rest of the MSF team arrived. The second doctor assumed the role of treatment leader, while the first one continued triage, re-assessment, and re-triage.

The complete medical team included, apart from the two doctors, three nurses who were checking vital signs, stabilizing patients, and administering the treatments and three Arabic interpreters who were used for communication and registration of patients. In addition, one psychologist was providing psychological first aid to patients recovering in the treatment area. Three MSF logisticians installed shadow nets, controlled entry to the treatment area, monitored the security situation during rioting, and helped with the patient transport to the treatment area on stretchers.

Entry or exit to the stadium was severely restricted due to overcrowding, lack of space, and poorly organized queues. An MSF team of two Farsi interpreters, one logistician, and a health promoter were blocked outside of the stadium, but they established an equipment and items supply line through a tiny window in the treatment area. In addition, they helped coordination with local Emergency Medical Services arriving for hospital referrals.

The single hospital of the island, part of the national health system, was located at a distance less than a kilometer away from the site. Nevertheless, only one ambulance was available in the local Emergency Medical Services to cover the complete Island of Kos, which included (in addition to refugees and tourists) a permanent local population of $33,000 .^{2}$

Three spontaneous medical responders among the refugees (one orthopedic surgeon and two medical students, all Syrians and English speakers) were used for monitoring of patients in the treatment area, under the supervision of the treatment leader MSF doctor, and also assisted with interpretation.

Simple Triage and Rapid Treatment (START) was used to guide assessment of patients during initial triage. ${ }^{3}$ Interventions were restricted to basic ones: maintaining airway, placement into recovery position under the shade, and transport to treatment area. No color tags were available and no visible tagging of patients took place; rather, the doctor orders were directly communicated to nurses, interpreters, and logisticians. A pocket pulse oximeter facilitated the triage.

In the treatment area, arterial blood pressure, temperature, and blood glucose measurements could be performed and a quick case history, clinical examination, and registration were taking place. Primary care and emergency drugs, wound dressing material, oropharyngeal airways, and ambu bag-valve-mask were available. Interventions consisted of: maintaining airway and placing in recovery position all collapsed victims; monitoring vital signs; keeping patients under the shade; passive cooling; oral rehydration with water for conscious patients and intravenous fluids infusion (normal saline, dextrose 5\%) for those who were hypotensive and/ or unable to drink; high-energy food bars (a couple of patients mentioned not having food for up to two days); non-steroidal anti-inflammatory drugs; wound cleaning; and bleeding control for minor injuries.

\section{Methods}

A description of the event in the form of a case report was prepared. Data review of the cases registered in the MSF patient consultation book was performed. This included all patients examined during the event. Anonymized data were entered in a spreadsheet and descriptive statistics obtained by means of Numbers'09 software version 2.0 (Apple Inc.; Cupertino, California USA). Retrospectively, START triage was used as a basis to categorize the diagnosed cases in green (minor), yellow (delayed treatment), and red (immediate treatment) categories.

The qualitative part included separate, private, confidential, unstructured interviews with each one of the three MSF Arabic language interpreters who worked inside the stadium during the event. The interviews took place a couple of days after the event. Interpreters were professional Arabic language interpreters employed as MSF staff and were not related in any way to the refugee population. The technique entailed the use of open-ended questions which focused on the difficulties they had faced during the response and what could have been done better.

Furthermore, a review of the literature in PubMed (National Center for Biotechnology Information, National Institutes of Health; Bethesda, Maryland USA) was organized using the $\mathrm{MeSH}$ terms "communication barriers," "triage," and "refugees" in order to identify the existence or not of similar reports. ${ }^{4}$ The literature search took place with a cutoff date of June 26th, 2016.

This was a descriptive and evaluative case report based on retrospective analysis of routinely collected, anonymized clinical data. This research was conducted in compliance and according to the MSF Research Ethics Framework Guidance Document (November 2013). ${ }^{5}$ The subjects of the research (interpreters) provided consent and their interviews were kept confidential.

\section{Results}

For the literature search with the terms "communication barriers AND triage AND refugees," no items were found in the PubMed database. The search "refugees AND triage" resulted in 23 articles while "communication barriers AND triage" resulted in another 58 articles. Among these 81 reviewed articles, no previous reports related to the use of interpreters in triage during a mass-gathering event involving refugees were identified (detailed lists of the literature search results with the reviewed articles are included as Supplementary Material; available online only.)

The MSF medical teams were inside the stadium for eight hours (11:00AM to 7:00PM). In total, 49 patients ( 42 men, seven women) were examined, the majority of whom were during the first few hours. Concerning their origin, 46 reported coming from Syria and three from Iraq. Age ranged from a 4-month-old baby to a 52-year-old adult with an average age of 25 years $($ mode $=22$; median $=25)$. During data review, $20(41 \%)$ patients were tagged green, including 12 with minor injuries. The rest consisted of one 
patient who lost his heart medications, one heat exhaustion case, a poorly breast-fed baby, one upper respiratory infection, one nose bleed, one patient with diarrhea, a 5-year-old with anorexia, and a patient with chronic complaints related to a past peripheral vascular surgery in the lower limb.

Another $11(22 \%)$ patients were tagged yellow, including five with heat exhaustion, two patients with nose bleeding, a hypertensive crisis (oral amlodipine was given), a diabetic with hyperglycemia, a pregnant woman with abdominal pain, and a peptic ulcer disease exacerbation (omeprazole was given). The remaining 18 (37\%) patients were tagged red, the majority due to their mental status since they could not follow simple commands or had lost consciousness. These included 16 heat syncope cases, a known epileptic who had seizures (diazepam intramuscularly was given), and a known ischemic heart disease patient with acute chest pain (aspirin was given).

Total hospital referrals among those 49 patients mentioned above included four patients: one heat syncope case, the abdominal pain during pregnancy case, the one who had epileptic seizures, and the patient with acute chest pain. There were no fatalities among the patients examined.

From the feedback of the MSF interpreters during the interviews, several constrains were identified. During the event, patients and bystanders appeared anxious, panicked, and psychologically stressed. They often shouted and talked without paying attention to the interpretation. Interpreters were continuously disturbed while performing their job by several different refugees addressing them simultaneously. Bystander refugees were collecting quickly around the interpreters once they realized they could speak their language. Interpreters were easily identifiable as they were wearing an MSF logo. This slowed them down further while moving through the crowd.

The team of three Arabic interpreters was shared by two doctors, three nurses, and one psychologist, often working at a distance of tens of meters from each other in an over-crowded and potentially unsafe environment. Communication was difficult between the team members. Due to background noise, one had to be standing face to face in order to be comprehended. Use of mobile phones was difficult due to background noise and areas of poor cellular network signal reception. Often, there was no visual contact, thus preventing any communication by gestures. In addition, medical staff were constantly moving, changing locations depending on the needs.

Most of the time, interpreters were not coupled with a designated doctor or a nurse but they shifted all the time depending on the needs. This created further confusion. Both doctors and nurses interrupted them often in order to claim their service from each other. Interpreters had no experience in triage settings, felt psychologically stressed, and had difficulties to prioritize and re-prioritize continuously where and for whom they would interpret as demand for their service exceeded their number.

\footnotetext{
Discussion

Language barrier is a well-documented reason for poor utilization of Emergency Medical Services. ${ }^{6,7}$ Inability to communicate may complicate the work of emergency medical teams and may create stress both to themselves as well as to the patients. During the ODRA (Poland) flood in 1997, communication difficulties due to different language between German and Polish people led to longer reaction times in disaster response. ${ }^{8}$
}

An interpreter may be well-trained to provide medical interpretation in the clinic setting or even the emergency department of a hospital. Providing the same service in the prehospital setting, especially in volatile or rapidly changing security conditions, might require a different set of skills and training. Interpretation may be even more important in case of medical patients. For them, an accurate case history and symptoms description is usually necessary for the diagnosis and treatment. By contrast, in certain trauma patients, the pattern of injury may be obvious from physical examination alone or it may be impossible to elicit a history in severe trauma when consciousness is impaired.

During this mass-gathering event, although there was a gap in interpreter management and allocation, when conflicts arose, the needs of the doctors in the team were respected as a priority. When no interpreter was available, doctors and nurses were trying to communicate with patients and bystanders in English or by gestures. Often, decisions were based on vital signs, clinical examination, as well as the overall impression of the doctor. ${ }^{9}$

Occasionally, bilingual refugees present on the scene were used as ad hoc interpreters. Nevertheless, it has been shown by Flores et al that "professional interpreters result in significantly lower likelihood of errors of medical interpretation than ad hoc interpreters and no interpreters at all." Furthermore, an interesting point is that "among professional interpreters, hours of previous training, but not years of experience, were associated with reduced interpretation errors." 10

One approach used in this mass-gathering event by the MSF medical team proved very useful. It consisted of directing the interpreters to transmit, independently of the presence of a doctor or nurse, simple emergency health education messages applicable to most patients self-presenting or brought by others, undergoing triage or treatment. Examples of messages included directions to bystanders for placing unconscious patients in recovery position and keeping them nil by mouth, giving water only to fully alert sitting patients to avoid aspiration, as well as explaining to anxious patients and bystanders the need to calm down and avoid hyperventilation which can cause "dizziness." Furthermore, one interpreter was designated permanently to the treatment area, one to the triage area, and one was shifting in between supporting the teams.

During the last years, there is an increase in numbers of refugees and immigrants arriving in Europe. ${ }^{11}$ This is the first report on the use of interpreters and its constrains during emergency medical care in a refugee mass-gathering incident in Europe.

Prior planning of reception systems should include the provision of adequate interpretation service since this improves both the outcome and patient's satisfaction. ${ }^{12,13}$ Patients who are refugees often have experienced psychological and physical trauma in their countries of origin or during their trip. History and physical examination facilitated by an interpreter, if needed, is important. ${ }^{14}$ On the other hand, lack of an interpreter may lead to unnecessary, costly, and harmful interventions in emergency medical care. ${ }^{15,16}$ Refugee reception facilities might be more vulnerable in case of a mass-gathering or mass-casualty incident since the language barrier will complicate any activities or medical aid undertaken in the event. Disaster preparedness planning should also include interpreters to address the needs of those persons not speaking the local language. ${ }^{17}$

Appropriate training might be needed as interpreters may not be familiar with triage and disaster-response concepts and the recommended actions in a given setting. Training in basic first aid 
and Basic Life Support might provide the necessary skills for an (accidentally isolated from his team) interpreter to be able to provide some basic care. Training in triage principles and techniques might facilitate coordination with the medical team in relevant settings. In order to answer these questions, further research is needed.

\section{Limitations}

This report is a simple case report and there are several limitations inherent to this type of research. It is based on the description of a single mass-gathering event which might not be representative or typical of this kind of events. Also, the patterns observed might not be applicable in a different context or different location. Retrospective evaluation of data after an event takes place may lead to more bias compared to a prospective study.

\section{References}

1. Spindler W. "Numbers of refugee arrivals to Greece increase dramatically." Briefing Notes, UNHCR; August 18, 2015. http://www.unhcr.org/55d3098d6.html. Accessed December 2, 2015.

2. EETAA (Hellenic Company of Local Development and Local Government). "Municipalities in Numbers." Athens, Greece; May 2013. Population data from 2011 census in Greece (in Greek language). http://web3.eetaa.gr:8080/ekdoseis/pdf/ 156.pdf. Accessed January 14, 2017.

3. Kahn C, Brooke Lerner E, Cone D. Chapter 12: Triage. In Koenig, Schultz. Disaster Medicine: Comprehensive Principles and Practices. New York USA: Cambridge University Press; 2010: 175-176.

4. National Center for Biotechnology Information, National Institutes of Health Bethesda, Maryland USA. PubMed Web site. http://www.ncbi.nlm.nih.gov/pubmed. Accessed January 14, 2017.

5. Médecins Sans Frontières. MSF Research Ethics Framework Guidance Document November 2013. http://fieldresearch.msf.org/msf/handle/10144/305288. Accessed January 14, 2017.

6. Subramaniam MR, Mahajan PV, Knazik SR, Giblin PT, Thomas R, Kannikeswaran N. Awareness and utilization of Emergency Medical Services by limited English proficient caregivers of pediatric patients. Prehosp Emerg Care. 2010; 14(4):531-536.

7. Smith MA, Lisabeth LD, Bonikowski F, Morgenstern LB. The role of ethnicity, sex, and language on delay to hospital arrival for acute ischemic stroke. Stroke. 2010; 41(5):905-909.

8. Benner T, Schaechinger U, Nerlich M. Medical telematics in disaster response. Stud Health Technol Inform. 2003;97:15-23.

\section{Conclusion}

Interpreters working as cultural mediators help to overcome the language and communication barriers between patients and the medical team. They are necessary for a proper refugee reception system and they should also be included in adequate numbers in authorities' planning when mass gatherings of refugees or migrants are anticipated.

Appropriate training may be needed for interpreters to develop skills useful in mass-gathering settings for a better coordination with the medical team. A potential question for further research is whether training in first aid, triage, and disaster-response concepts with a curriculum specifically designed and adapted for interpreters could improve their efficiency and coordination with the medical team in mass gatherings and similar prehospital settings.

\section{Supplementary Material}

To view supplementary material for this article, please visit https://doi.org/10.1017/S1049023X17006781

9. Kulla M, Josse F, Stierholz M, Hossfeld B, Lampl L, Helm M. Initial assessment and treatment of refugees in the Mediterranean Sea (a secondary data analysis concerning the initial assessment and treatment of 2656 refugees rescued from distress at sea in support of the EUNAVFOR MED relief mission of the EU). Scand J Trauma Resusc Emerg Med. 2016;24(1):75.

10. Flores G, Abreu M, Barone CP, Bachur R, Lin H. Errors of medical interpretation and their potential clinical consequences: a comparison of professional versus ad hoc versus no interpreters. Ann Emerg Med. 2012;60(5):545-553.

11. UNHCR. Europe's refugee emergency response update \# 12. November 20-26, 2015 http://data.unhcr.org/mediterranean/regional.php. Accessed January 14, 2017.

12. Flores $\mathrm{G}$. The impact of medical interpreter services on the quality of health care: a systematic review. Med Care Res Rev. 2005;62(3):255-299.

13. Mahmoud I, Hou XY, Chu K, Clark M, Eley R. Satisfaction with emergency department service among non-English-speaking background patients. Emerg Med Australas. 2014;26(3):256-261.

14. Crosby SS. Primary care management of non-English-speaking refugees who have experienced trauma: a clinical review. JAMA. 2013;310(5):519-528.

15. Tate RC, Kelley MC. Triage in the Tower of Babel: interpreter services for children in the prehospital setting. Pediatr Emerg Care. 2013;29(12):1280-1282.

16. Hampers LC, Cha S, Gutglass DJ, Binns HJ, Krug SE. Language barriers and resource utilization in a pediatric emergency department. Pediatrics. 1999;103 (6 Pt 1):1253-1256.

17. Shiu-Thornton S, Balabis J, Senturia K, Tamayo A, Oberle M. Disaster preparedness for limited English proficient communities: medical interpreters as cultural brokers and gatekeepers. Public Health Rep. 2007;122(4):466-471. 